\title{
Determination of the radon concentration in homes depending on the insulation used for the floor
}

\author{
Andreea Cristina Tataru ${ }^{1 *}$, Aurora Stanci $^{2}$, and Dorin Tataru ${ }^{1}$ \\ ${ }^{1}$ University of Petrosani, Department of Mechanical and Industrial Engineering and Transports, \\ Universitatii street, No.20, Petrosani, Romania \\ ${ }^{2}$ University of Petrosani, Departament of Management, Environmental Engineering and Geology, \\ Universitatii street, No.20, Petrosani, Romania
}

\begin{abstract}
A current environmental problem is the presence of radon inside the house. Radon (222Rn) is a noble, colourless and odourless gas that comes from radioactive elements naturally present in rocks and soil. Being gas, it is released from the ground with a tendency to concentrate in closed spaces such as caves, mines, cellars but also in any rooms in the basement, ground floor or with poor ventilation. Due to the differences between the temperature inside and the soil, the radon in the soil will move naturally to the interior of the houses, concentrating in closed rooms. The accumulation of radon inside buildings is a consequence of technological progress. Insulation work, tightly closed windows, poor ventilation of rooms lead to unobservable increase in radon concentration inside. In this paper we aim to present the values of the concentration of radon accumulated inside a house depending on the way of insulating the floor.
\end{abstract}

\section{Introduction}

One of the newest environmental problems is radon pollution.

In principle, any house, whether old or new, with or without a basement, even well insulated can have problems with this type of radioactive gas. Radon is radioactive and therefore carcinogenic. Radon is also called the "silent killer."

Radioactivity is everywhere in our environment and can thus be detected in the water we drink, in the food we ingest, in the air we breathe. Ionising radiation can have artificial or natural origins. [1]. By far, natural radiation is the main contributor to the total dose since less than $15 \%$ of the radiation we receive is man-made, from which $98 \%$ come from medical activities (diagnostics and therapy) (Figure 1).

In most circumstances, the naturally radioactive gas called radon (RnP 222 isotope with a half-life of 3.8 days) is the major contributor to the total dose. This gas is produced by the radioactive decay of uranium which is an element that is naturally present in the earth's crust. Radon transports radioactivity mainly by diffusion mechanisms through the ground, and its concentration in our environment thus depends mainly on geological factors, soil

${ }^{*}$ Corresponding author: andreeastanci@yahoo.com 
texture, soil water content, and the pressure difference between the gas in the soil and at the surface.

Relatively heavier than air, radon shows low concentrations outdoor but tends to be trapped in basements and the lower floors of buildings. For the same reasons, it is often found at high concentrations in mining galleries. Because radon is considered to be the main contributor to lung cancer second to smoking, the European Commission constantly makes efforts to inform the public about this natural hazard. Recent studies [2] have shown that radon in homes causes about 20,000 lung cancer deaths in the European Union (EU) each year. This is about $9 \%$ of the total lung cancer deaths in the EU and about $2 \%$ of cancer deaths overall.

Studies show that in Romania, there are 16 counties where the level of radon concentration can cause problems in homes. In addition, building materials purchased in these areas may be more contaminated than in other areas.

The 16 counties are Alba, Arad, Bihor, Bistriţa-Năsăud, Braşov, Caraş-Severin, Cluj, Covasna, Harghita, Hunedoara, Maramureş, Mureş, Sibiu, Sălaj, Satu Mare and Timiş. Transylvania, in general, is the area most exposed to radon increases in the air, but also the large urban agglomerations: Bucharest, Iasi, Timisoara, Constanta and Brasov. [3], [4], [5]

\section{Radon}

Radon is an odourless, colourless radioactive gas that is formed by the radioactive decay of uranium from the earth. Although it cannot be seen or smelled, radon is present in all homes, in various concentrations, and is considered a cause for lung cancer.

From a scientific point of view, radon is a chemical element with the symbol Rn and atomic number 86. It was discovered in 1900 by Friedrich Ernst Dorn and is part of the rare gas group.

The increase in the concentration of radon in the atmosphere announces the approach of an earthquake. Radon is odourless, colourless, tasteless, but at low temperatures below freezing $\left(-71^{\circ} \mathrm{C}\right)$ it has a bright phosphorescent colour. Radon is found in soil, rocks and water and cannot be detected by humans, but it enters the house from the foundation and through the water well.

Starting with 2017 in Romania, the radon concentration came under the attention of those from the National Commission for the Control of Nuclear Activity. (CNCAN). They have developed several documents that provide for the determination of radon concentration and the maximum allowable limits. A National Radon Action Plan was also drawn up in Romania by CNCAN. Within this National Radon Action Plan is also the creation of a map of radon radiation. [3], [4], [5]

\section{The presence of radon in homes}

Radon in homes requires special attention because its presence beyond certain limits can affect life. All homes are affected by radon. Radon concentrations can vary widely.

There are certain factors that can affect the radon concentration inside.

- soil characteristics: the radon concentration is dependent on the uranium content in the soil, respectively on the soil permeability.

- type of construction: the way the house is designed, the contact surface with the ground, the presence of cracks in the walls or the floor affect the radon concentration inside.

- lifestyle: frequent ventilation of rooms, the use of air conditioning units have a strong impact in reducing the concentration of radon inside. 
- climate: weather variations (temperature, wind, humidity, pressure) can affect the amount of radon that migrates into the air in homes.

Because the concentration of radon inside depends on many factors, the only way we can find out the level we are exposed to is to monitor the level of radon in the house.

In this paper we aimed to study the concentration of radon in the home depending on the insulation used.

For this study, we performed radon monitoring in a house where different materials were used to insulate the floor.

The monitored house is located on the administrative territory of Lupeni Municipality, Hunedoara County. (Fig.1)

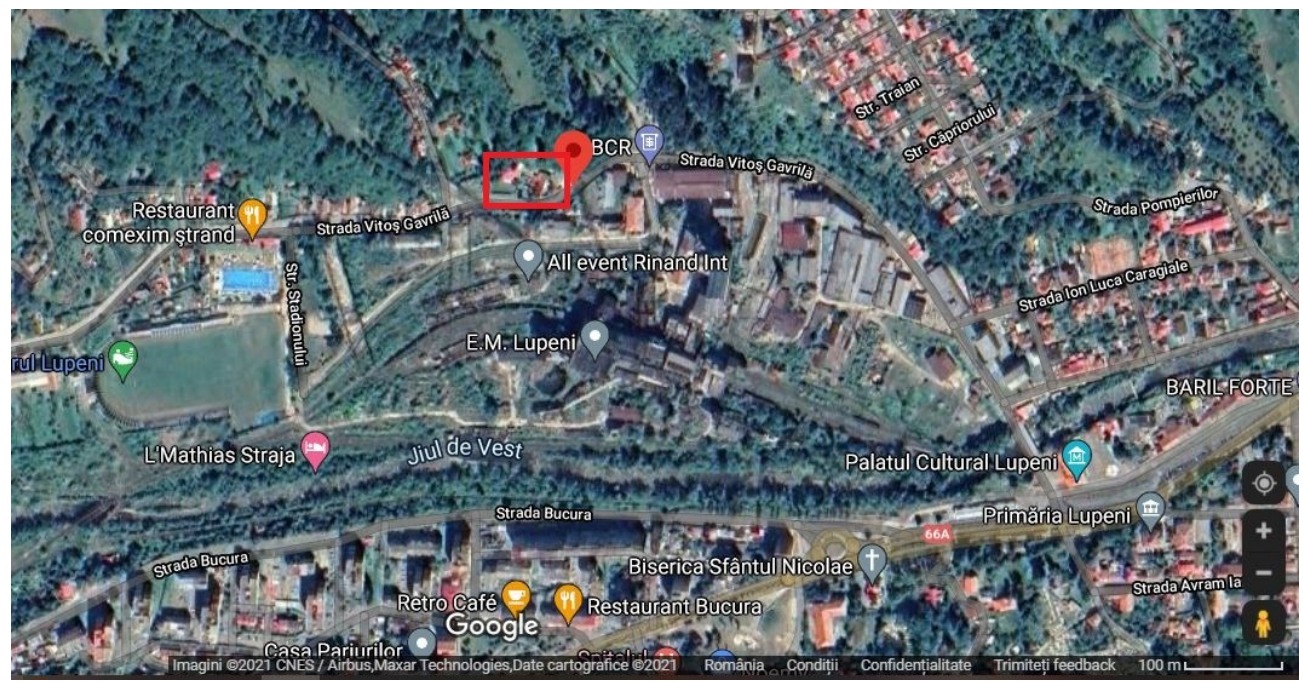

Fig. 1. Location of the house where the radon monitoring was performed

The monitoring was carried out in three different rooms, completely separate, over a period of three months.

Each room has a different type of floor insulation as follows.

- Room 1:

- $10 \mathrm{~cm}$ reinforced concrete

- Room 2:

- $15 \mathrm{~cm}$ reinforced concrete B150,

- underfloor heating insulation QTEQ EPS 100/25 m with aluminized foil $2 \mathrm{~cm}$ thick, the thickness of the aluminium foil is $0.1 \mathrm{~mm}$

- $\quad 7 \mathrm{~cm}$ semi-medium screed.

- Room 3:

- $15 \mathrm{~cm}$ reinforced concrete B150,

- extruded polystyrene $2 \mathrm{~cm}$,

- underfloor heating insulation QTEQ EPS 100/25 m with aluminized foil $2 \mathrm{~cm}$ thick, the thickness of the aluminium foil is $0.1 \mathrm{~mm}$

- $7 \mathrm{~cm}$ semi-medium screed. 


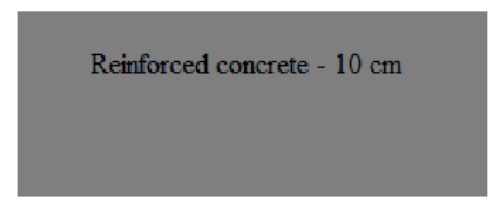

Room no. 1

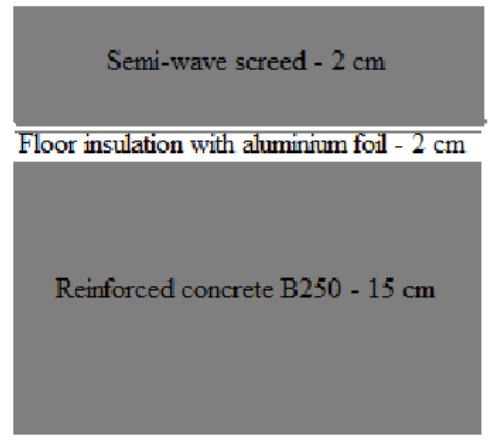

Room no. 2

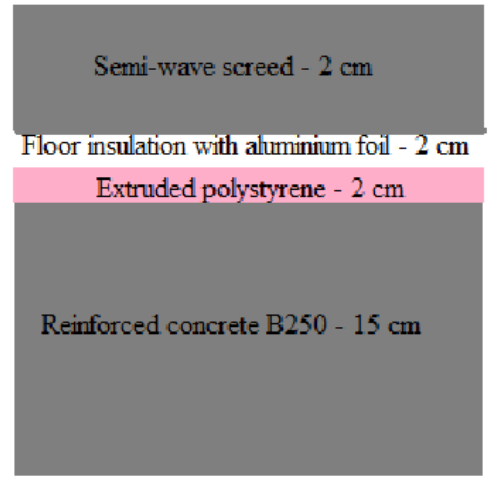

Room no. 3

Fig. 2. Isolation used in the three rooms in which radon monitoring was performed

To determine the level inside the house, the radon concentration in the indoor air was measured. A normal value of the radon concentration in the room must not exceed $100 \mathrm{~Bq} /$ $\mathrm{m}^{3}$. If the recorded values are between 100 and $300 \mathrm{~Bq} / \mathrm{m}^{3}$, rapid solutions to reduce the radon concentration must be found. The maximum permissible limit of the radon concentration according to CNCAN norms is $300 \mathrm{~Bq} / \mathrm{m}^{3}$.

Radon was measured using the radon detector Airthings Wave Plus This device can parametrically measure the air inside a room. Airthings Wave offers detailed information about indoor air quality, through the 6 built-in sensors:

- $\quad$ sensor for measuring radon concentration

- ambient temperature sensor

- relative air humidity sensor

- atmospheric pressure sensor

- sensor for measuring $\mathrm{CO} 2$ concentration

- $\quad$ sensor for measuring TVOC (volatile organic components)

The results obtained from the measurements are presented in table 1.

A high radon value can be observed in chamber 1. During the period when the chamber was not ventilated, the radon concentration reached values of $237 \mathrm{~Bq} / \mathrm{m}^{3}$. These values are due to the fact that the floor of room 1 has only a $10 \mathrm{~cm}$ layer of concrete. This layer of concrete was poured with the renovation of the house in 1972 and has cracks.

In chambers 2 and 3 the radon value is much lower, the maximum values reaching only $92 \mathrm{~Bq} / \mathrm{m}^{3}$ for chamber 2 and $64 \mathrm{~Bq} / \mathrm{m}^{3}$ for chamber 3 . These values are given by the existence of cracks in the floor that allow radon to penetrate.

The much lower values of radon in chambers 2 and 3 are also due to the insulation used. In both rooms was used QTEQ EPS floor heating insulation 100/25 m with drawn aluminized foil and adhesive tape, the thickness of the aluminium foil is $0.1 \mathrm{~mm}$. 
In the case of room 3 the values are the lowest. Here, $2 \mathrm{~cm}$ extruded polystyrene was also used to insulate the floor.

The daily radon concentration values recorded in the last month for each chamber are shown in Figures 3, 4 and 5.

Table 1. Radon concentration values for the three monitored chambers

\begin{tabular}{|c|c|c|c|}
\hline Location & $\begin{array}{c}\text { Maximum radon } \\
\text { concentration }\left[\mathbf{B q} / \mathbf{m}^{\mathbf{3}}\right]\end{array}$ & $\begin{array}{c}\text { Minimum Radon } \\
\text { Concentration } \\
{\left[\mathbf{B q} / \mathbf{m}^{3}\right]}\end{array}$ & $\begin{array}{c}\text { Average radon } \\
\text { concentration }\left[\mathbf{B q} / \mathbf{m}^{\mathbf{3}}\right]\end{array}$ \\
\hline Room no. 1 & 237 & 38 & 117 \\
\hline Room no. 2 & 92 & 8 & 48 \\
\hline Room no. 3 & 64 & 0 & 24 \\
\hline
\end{tabular}

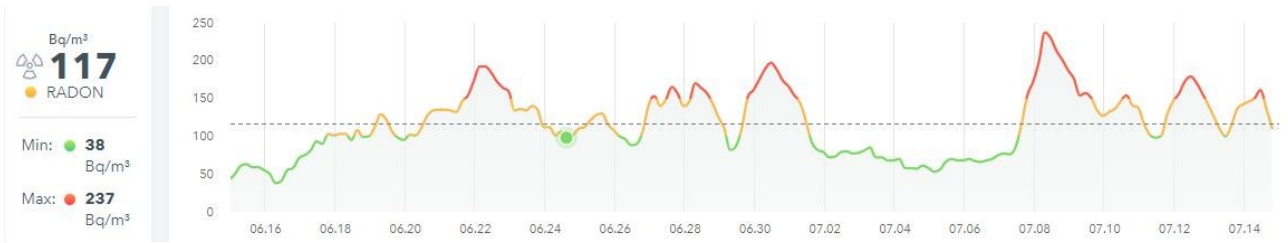

Fig. 3. Radon concentration for the period 14.06.2021 - 14.07.2021 for room 1

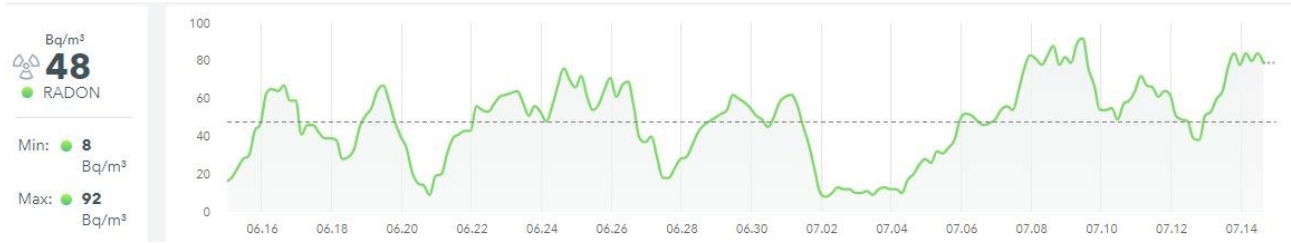

Fig. 4. Radon concentration for the period 14.06.2021 - 14.07.2021 for room 2

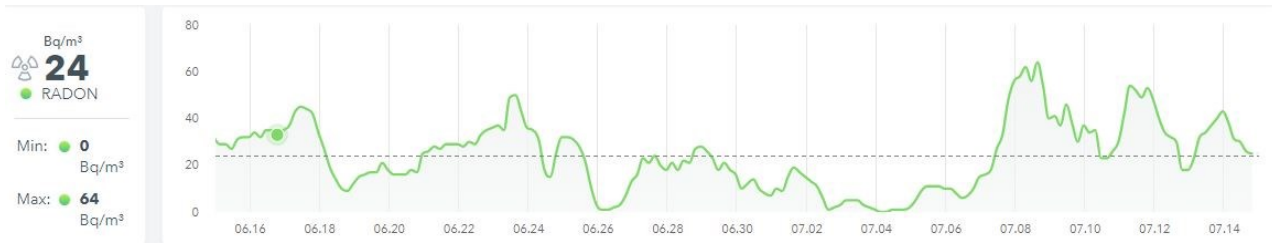

Fig. 5. Radon concentration for the period 14.06.2021 - 14.07.2021 for room 3

The only room that presents a risk of radiation is room 1 . The average value of the values recorded for this room, namely $117 \mathrm{~Bq} /{ }^{3}$, reaches the warning level. The maximum values recorded reach the danger level. In the case of room 1 it can be seen that a renovation of the floor is necessary to reduce the radon level inside the room.

As a measure to reduce the level of radon for room 1 we can mention the sealing of cracks in floors and walls and the use of methods of insulating the floor.

In the case of rooms 2 and 3, which have already been renovated, frequent ventilation of the radon level inside the rooms is sufficient.

\section{Conclusions}

Radon is an important source of air pollution, as it is a radioactive gas that can cause serious health problems. Radon is found in rocks. 
Radon reaches the inside of homes through cracks in the floor.

Depending on how the floor is insulated, the concentration of radon inside the house differs.

In the case of a concrete floor of $15 \mathrm{~cm}$, old and with cracks, the radon concentration in the room is very high, the average of the results obtained when monitoring over a period of 3 months being $117 \mathrm{~Bq} / \mathrm{m}^{3}$. This value reaches the warning threshold.

In the case of rooms renovated and insulated with extruded polystyrene $2 \mathrm{~cm}$, and underfloor heating insulation QTEQ EPS 100 with aluminized foil, the value of radon concentration is low.

\section{References}

1. M. Charles, UNSCEAR Report 2000: Sources and Effects of Ionizing Radiation. Journal of Radiological Protection, (2001).

2. S. Darby, D. Hill, A. Auvinen, J. M Barros-Dios, H. Baysson, F. Bochicchio, H. Deo, R. Falk, F. Forastiere, M. Hakama, I. Heid, L. Kreienbrock, M. Kreuzer, F. Lagarde, I. Mäkeläinen, C. Muirhead, W. Oberaigner, G. Pershagen, A. Ruano-Ravina, E. Ruosteenoja, A. Schaffrath Rosario, M. Tirmarche, L. Tomásek, E. Whitley, H.-E. Wichmann and R. Doll. Radon in homes and risk of lung cancer: collaborative analysis of individual data from 13 European case-control studies. (2005)

3. A. C. Tataru, A. Stanci, Determination of Radon Level in Existing Buildings and Remedial Actions, The 19th edition - „Modern Technologies for the 3rd Millenium”, ISBN 978-8887729-61-0, (2020)

4. A.C. Tataru, A. Stanci, Monitoring the level of radioactive pollution of the dumps and ponds in the Jiu Valley, Ecoterra - Journal of Environmental Research and Protection, ISSN 2248-3128, (2019)

5. C. Cosma*, D. Ciorba, A. Timar, K. Szacsvai, Al. Dinu, Radon exposure and lung cancer risk in Romania (2005) 